\title{
Functional near-infrared spectroscopy (fNIRS) of posterolateral cerebellum and prefrontal cortex for fNIRS-driven cerebellar tES
}

Shubh Mohan Singh

PGIMER Chandigarh

Kavya Narendra Kumar

University at Buffalo SUNY, USA

Pushpinder Walia

University at Buffalo SUNY, USA

Shashi Ranjan

IIT Gandhinagar, India

Zeynab Rezaee

University at Buffalo SUNY, USA

Uttama Lahiri

IIT Gandhinagar, India

Anirban Dutta ( $\nabla$ anirband@buffalo.edu )

University at Buffalo SUNY, USA

\section{Method Article}

Keywords: cerebellar tES, cerebellar cognitive affective/Schmahmann syndrome, fNIRS, neuroimagingguided tES

Posted Date: July 16th, 2021

DOl: https://doi.org/10.21203/rs.3.rs-725723/v1

License: (a) (1) This work is licensed under a Creative Commons Attribution 4.0 International License. Read Full License 


\title{
Functional near-infrared spectroscopy (fNIRS) of posterolateral cerebellum and prefrontal cortex for fNIRS-driven cerebellar tES - a case report
}

\author{
Shubh Mohan Singh, Kavya Narendra Kumar, Pushpinder Walia, Shashi Ranjan, Zeynab Rezaee, \\ Uttama Lahiri, and Anirban Dutta
}

\begin{abstract}
Cerebellar transcranial direct current stimulation (ctDCS) has been shown to facilitate standing balance in stroke survivors where a good general linear model fit was found in the latent space between the mean lobular ctDCS electric field strength with the oxy-hemoglobin concentrations (HbO) from functional near-infrared spectroscopy (fNIRS) and $\log 10$ transformed electroencephalogram (EEG) bandpower at the prefrontal cortex (PFC) and the sensorimotor cortex in the responders. Recent works have also found that the infra-slow activity $(0.01-0.10 \mathrm{~Hz})$ and delta band $(0.5-4 \mathrm{~Hz})$ activity propagated in opposite directions between the cerebellum and cerebral cortex. Therefore, in this study, we tested the feasibility of fNIRS of cerebellum and PFC where infra-slow $(0.01-0.10 \mathrm{~Hz})$ PFC HbO activity was used to drive (phase amplitude coupling) $4 \mathrm{~Hz}$ cerebellar transcranial alternating current stimulation (ctACS) at right lobules VI-CrusI/II-VIIb. We found that $2 \mathrm{~mA}$ ctDCS evoked similar $\mathrm{HbO}$ response across cerebellum and PFC brain regions $(\alpha=0.01)$; however, $2 \mathrm{~mA}$ ctACS evoked HbO across brain regions that was statistically different $(\alpha=0.01)$.

Clinical Relevance-We showed the feasibility of fNIRS of cerebellum and PFC, and fNIRS-driven ctACS at $4 \mathrm{~Hz}$ that may facilitate cognitive function via the frontoparietal network in cerebellar cognitive affective/Schmahmann syndrome.
\end{abstract}

\section{INTRODUCTION}

Middle cerebral artery (MCA) stroke is the most common stroke where many survivors do not have the involvement of the lower limb cortical area unless deep territory of the MCA is involved. In these subjects, the medial part of the frontal and the parietal lobe are spared that are supplied by the anterior cerebral artery (ACA). Furthermore, cerebellum supplied by the cerebellar arteries is spared along with the cerebello-cerebral connectivity to the medial part of the frontal lobe when deep territory of the MCA is not involved. Such stroke survivors have been found to be mostly responsive to cerebellar transcranial direct current stimulation (ctDCS) for standing balance rehabilitation [1] where functional near-infrared spectroscopy (fNIRS) can be used to identify responders [2]. This is relevant since contra-lesional ctDCS showed promise for improving standing balance performance in prior works [3],[4]. We also found a good general linear model (GLM) fit between the first principal component of the mean lobular ctDCS electric field strength

\footnotetext{
*Research supported by the Indian Ministry of Human Resource Development (MHRD)'s Scheme for Promotion of Academic and Research Collaboration (SPARC), grant number 2018-2019/P721/SL. Authors have no potential conflicts of interest.

S.M. Singh is with the PGIMER, Chandigarh, India.

K.N. Kumar is with the University at Buffalo SUNY, USA.
}

with the latent variables of oxy-hemoglobin concentrations (HbO) from fNIRS and log10-transformed electroencephalogram (EEG) bandpower at the prefrontal cortex (PFC) and the sensorimotor cortex in the responders [2]. Here, a recent work found that the frontoparietal network is disproportionately expanded in the cerebellum compared to cortex [5]. Furthermore, Marek et al. [5] found that cerebellar blood-oxygen-level-dependent imaging signals temporally lag the cortex where the infra-slow activity $(0.01-0.10 \mathrm{~Hz})$ and delta band $(0.5-4 \mathrm{~Hz})$ activity propagated in opposite directions between the cerebellum and cerebral cortex. In principal accordance, we conducted feasibility testing of fNIRS in young and healthy subjects to drive (phase amplitude coupling) cerebellar transcranial alternating current stimulation (ctACS) at $4 \mathrm{~Hz}$ using infra-slow $(0.01-$ $0.10 \mathrm{~Hz}$ ) PFC HbO activity. Here, fNIRS-driven $4 \mathrm{~Hz}$ ctACS is expected to facilitate cerebellar brain inhibition [6] better than ctDCS that we measured using fNIRS [2].

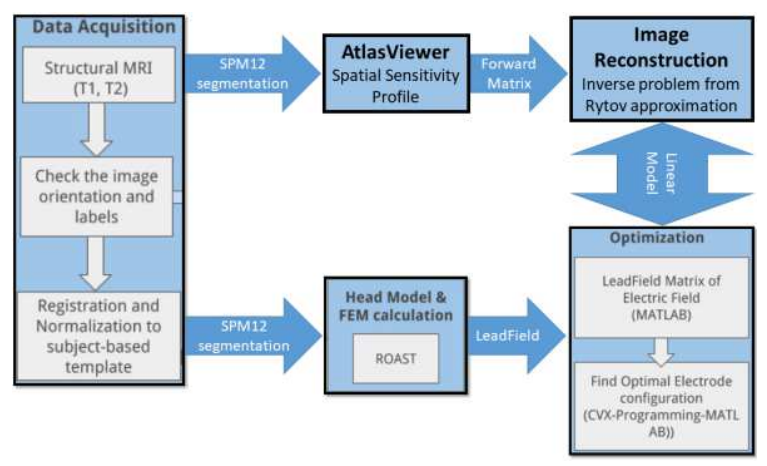

Fig. 1: Neuroimaging guided transcranial electrical stimulation

\section{METHODS}

\section{A. fNIRS sensitivity profile for neuroimaging guided ctDCS}

Individual head model was created based on structural magnetic resonance images (MRI) from our prior work on Cerebellar Lobules Optimal Stimulation (CLOS) pipeline [7]. $\mathrm{MR}$ images were taken from a young and healthy volunteer in accordance with the Declaration of Helsinki - a statement of ethical principles for medical research involving humans study. The study was approved (IEC/2019-20/4/UL/046) at the

P. Walia is with the University at Buffalo SUNY, USA

S. Ranjan is with the Indian Institute of Technology Gandhinagar, India.

Z. Rezaee is with the University at Buffalo SUNY, USA.

U. Lahiri is with the Indian Institute of Technology Gandhinagar, India.

A. Dutta is with the University at Buffalo SUNY, USA (e-mail: anirband@buffalo.edu). 
Indian Institute of Technology Gandhinagar, India and University at Buffalo, USA.

Fig. 1. shows neuroimaging guided transcranial electrical stimulation pipeline using subject-specific head model from SPM12 segmentation algorithm (https://www.fil.ion.ucl.ac.uk/spm/software/spm12/) in Matlab (Mathworks Inc., USA). CLOS pipeline can use realistic volumetric-approach to simulate transcranial electric stimulation-ROAST - pipeline [8] (or, SimNIBS [9]) for finite element analysis of the electric field for electrode optimization [7],[4]. In our prior work, we have optimized bipolar ctDCS montage for lower-limb motor representations and dentate nuclei in case of stroke survivors [4]. Here, motor representations are twice whereas non-motor representations (attentional/executive and default-mode) are thrice in each cerebellar cortical hemisphere (lobules VI-Crus I; lobules Crus II-VIIB; lobules IX-X) [10]. Three functional domains were found in the cerebellar cortex, i.e., the functional gradients in cerebellum, where Crus I/II intersection is the intersection of first and the second default-mode representation [10]. Viral tracing studies in non-human primates have shown Crus I/II to have projections only to the prefrontal cortex [11]. Also, functional MRI studies have shown Crus I connectivity with the medial prefrontal cortex (MPFC) while Crus II with the dorsolateral prefrontal cortex (DLPFC) [12]. Therefore, in this study, we optimized ctDCS/ctACS montage for the non-motor representation in the right cerebellar hemisphere, namely, right lobules VI-CrusI/II-VIIb, using our CLOS pipeline [7].

We compared ctACS and ctDCS effect using the same montage based on fNIRS oxyhemoglobin concentration changes $(\mathrm{HbO})$ at the prefrontal cortex (PFC), and cerebellum (CER). Session consisted of a block design of 3 min rest and ctDCS/ctACS duration of 5 min which was chosen based on prior works that showed significant increase in the cortical excitability [13] and cerebral blood flow changes [14]. Also, our computational modeling [15] showed feasibility of model linearization for evaluating the acute effects during first 150 seconds of primary motor cortex tDCS in healthy humans using fNIRS based measure of blood volume. In this study, we used the SPM12 segmented headmodel to run the freely available Monte-Carlo photon transport software (tMCimg) in the AtlasViewer [16] to compute fNIRS sensitivity profile based on our prior work [17]. Then, AtlasViewer [16] determined $\mathrm{HbO}$ hemodynamic response function to ctDCS/ctACS where a GLM fit (Linear Model) is postulated.

\section{B. fNIRS data acquisition and processing}

Ten young and healthy right-handed subjects ( 2 females, age: 21-25 years) volunteered for this case report under IRB approval at the University at Buffalo, IIT Gandhinagar, and PGIMER, India. The session consisted of a block design of 2.5 min baseline, ctDCS/ctACS period of $5 \mathrm{~min}$, and $2.5 \mathrm{~min}$ postintervention measures. We postulated [15] that the immediate tDCS effects on blood volume is mainly due to the pial vessels (sensitive to fNIRS). In this study, fNIRS was conducted using NIRSPORT 2 (NIRx, USA) where our optode montage consisted of 12 long-separation $(\sim 3.5 \mathrm{~cm})$ sources, 3 longseparation detectors, and 3 short-separation $(<1 \mathrm{~cm})$ detectors that covered PFC, and CER. This long-separation optode montage was selected to match our low-channel count montage (Octamon+, Artinis Medical Systems, Netherlands) used in stroke study where fNIRS sources were positioned at AF7, AF3, AF8, AF4, CP4, FC4, CP3, and FC3, and the two detectors were placed at the $\mathrm{Cz}$ and $\mathrm{FPz}$ with a source-detector distance of around $35 \mathrm{~mm}$ [2]. For CER, fNIRS sources were positioned at the PO7, PO9, PO8, and PO10, and the detector was placed at Iz based on fNIRS Optodes' Location Decider (fOLD) [18].

Data processing was conducted using open-source HOMER3 toolbox [19] in Matlab (Mathworks Inc., USA). Raw optical intensity signal was first converted into optical density (function: hmrR_Intensity2OD), then motion artifact detection and correction was conducted using a hybrid method based on spline interpolation method and Savitzky-Golay filtering (function: hmrR_MotionCorrectSplineSG) [20] using default parameters. Then, bandpass filtering was conducted (function:hmrR_BandpassFilt:Bandpass_Filter_OpticalDensi ty) within $0.01-0.1 \mathrm{~Hz}$ followed by conversion to oxyhemoglobin ( $\mathrm{HbO})$ and deoxy-hemoglobin ( $\mathrm{HHb})$ concentration (function: hmrR_OD2Conc). Finally, hemodynamic response function (HRF) was computed using General Linear Model (GLM) (function: hmrR_GLM_new) with short separation regression performed with the nearest short separation channel. GLM determined the HRF during stimulation period from the resting state using ordinary least squares [21] with consecutive sequence of Gaussian functions $($ stdev $=0.5$, step $=0.5)$.

\section{(a)}
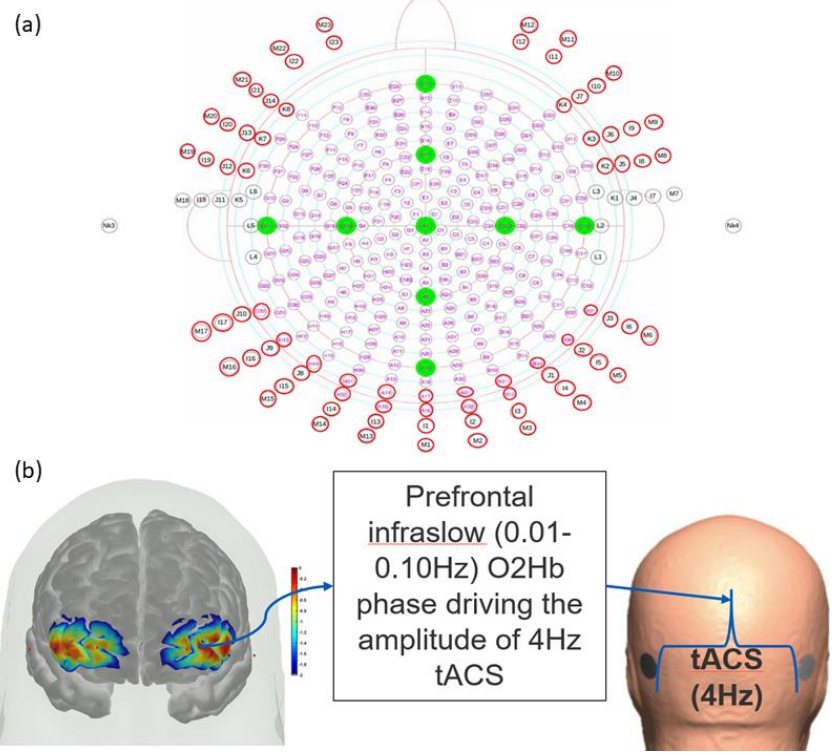

Fig. 2: CLOS optimization of ctDCS/ctACS electrode montage using standard EEG locations. (a) high-density electrode montage used for optimization. (b) phase of the infraslow $(0.01-0.10 \mathrm{~Hz})$ oxyhemoglobin $(\mathrm{O} 2 \mathrm{Hb})$ oscillations at the left $\mathrm{PFC}$ driving the amplitude of the $4 \mathrm{~Hz}$ cerebellar tACS optimized for the right lobules VI-CrusI/II-VIIb.

\section{Optimization of montage and fNIRS-driven ctACS}

We found [2] a linear relationship between electric field distribution and the $\mathrm{HbO}$ response using GLM analysis of variance (ANOVA) for the ctDCS montages where the montage for the dentate nuclei targeted Crus I/II more than the ctDCS montage targeting lower limb representations. In this study, we optimized the ctDCS/ctACS electrode montage for non-motor representation, i.e., right lobules VI-CrusI/II-VIIb, 
using EEG locations - Fig. 2a [4]. Amplitude of the $4 \mathrm{~Hz}$ ctACS with optimized montage was driven by the phase of the infraslow $(0.01-0.10 \mathrm{~Hz}) \mathrm{HbO}$ oscillations at left PFC found using Hilbert transform for the analytic signal using $60 \mathrm{sec}$ sliding window - Fig. 2b. The maximum tACS amplitude was set at $\pm 2 \mathrm{~mA}$ in the Starstim $8 \mathrm{tES}$ device (Neuroelectrics).

\section{RESULTS}

\section{A. CLOS optimization of ctDCS montage}

Fig. 3 shows our results from CLOS optimization for maximum electric field strength at the non-motor representation, right lobules VI-CrusI/II-VIIb [7], where $2 \mathrm{~mA}$ at OI2 and $-2 \mathrm{~mA}$ at E145 were found optimal (see Fig. 3c). Fig. 3a, b shows that the optimized electric field strength is high $(>0.2151 \mathrm{~V} / \mathrm{m})$ at CrusI/II-VIIb where the deep nuclei received comparable intensity (see Fig. 3d). Here, lobules VICrusI/II-VIIb (non-motor representations) and lobules VIIbIX (lower-limb motor representations) were successfully delineated in the posterior lobe of cerebellum.
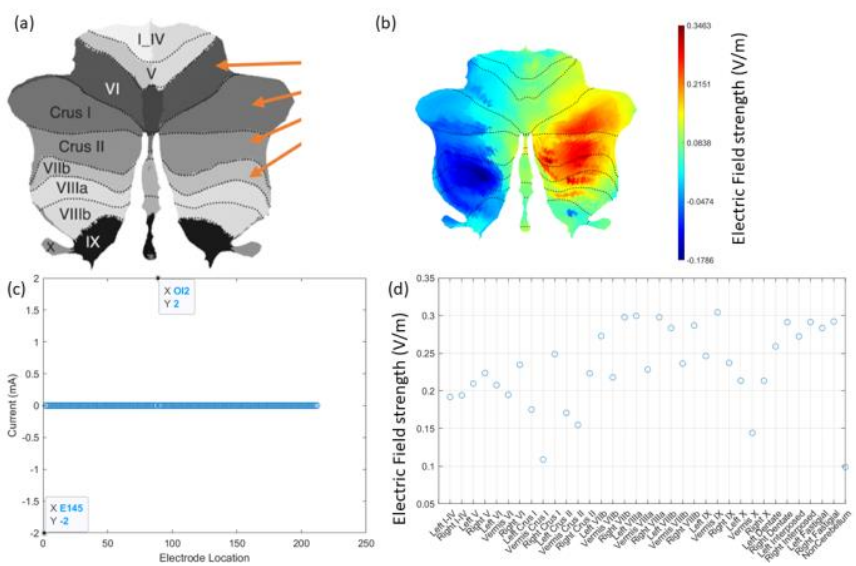

Fig. 3: CLOS optimization of ctDCS electrode montage for right lobules VI-CrusI/II-VIIb. (a) right lobules VI-CrusI/II-VIIb in SUIT flatmap. (b) optimized lobular electric field strength in SUIT flatmap. (c) optimized current ( $m A)$ at electrodes $(2 m A$ at OI2 and $2 m A$ at E145). (d) optimized lobular electric field strength $(\mathrm{V} / \mathrm{m})$ across lobules (note the electric field strength at the deep nuclei).

\section{B. fNIRS sensitivity profile}
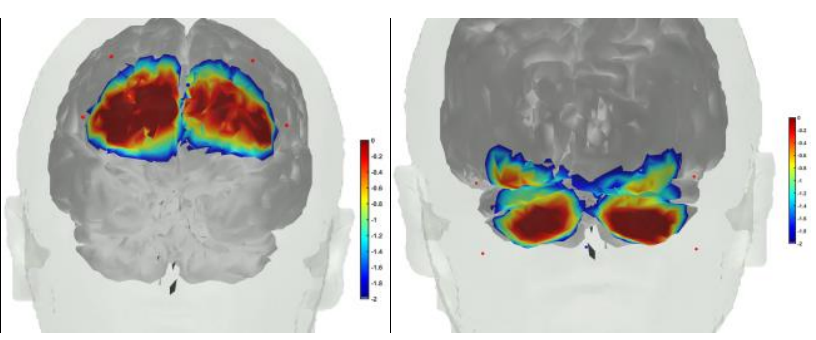

Fig. 4: Left panel - fNIRS sensitivity profile where sources were positioned at AF7, AF3, AF8, AF4, and detector placed at the FPz. Right panel - fNIRS sensitivity profile where sources were positioned at PO7, PO9, PO8, and PO10, and the detector was placed at the Iz.

Our PFC optode montage covered MPFC, and partly the DLPFC, as shown in the left panel of Fig. 4. Right panel of Fig. 4 shows the fNIRS sensitivity profile for CER optode montage (confirmed with fOLD [18]) where we found from our MRI-based headmodel in AtlasViewer that the fNIRS sensitivity was mainly at the Crus I/II of the cerebellum. This is important since Crus I/II intersection is the intersection of first and the second default-mode representation [10].

\section{C. fNIRS HbO response to ctDCS}

Fig. 5 shows the average $\mathrm{HbO}$ response at PFC and CER across subjects due to $2 \mathrm{~mA}$ ctDCS that led to an increase in the $\mathrm{HbO}$ at cerebellar hemispheres as well as a delayed $(>150 \mathrm{sec})$ increase in $\mathrm{HbO}$ at bilateral PFC. Fig. 6 shows the $\mathrm{HbO}$ response due to $2 \mathrm{~mA}$ ctACS where a small change in the $\mathrm{HbO}$ was found at the cerebellar hemispheres (fNIRS sensitive to Crus I/II only) while an overall decrease in the $\mathrm{HbO}$ was found at the bilateral PFC. However, in the right $\mathrm{PFC}, \mathrm{HbO}$ response started to increase after $150 \mathrm{sec}$ following initial decrease $(<150 \mathrm{sec})$. Left panel of Fig. 7 shows box-plot of post-intervention $\mathrm{HbO}$ change where $2 \mathrm{~mA}$ ctDCS evoked similar $\mathrm{HbO}$ change across brain regions $(\alpha=0.01)$. However, $2 \mathrm{~mA}$ ctACS evoked $\mathrm{HbO}$ change that was statistically ( $\alpha=0.01$ ) different across brain regions - right panel of Fig. 7 .
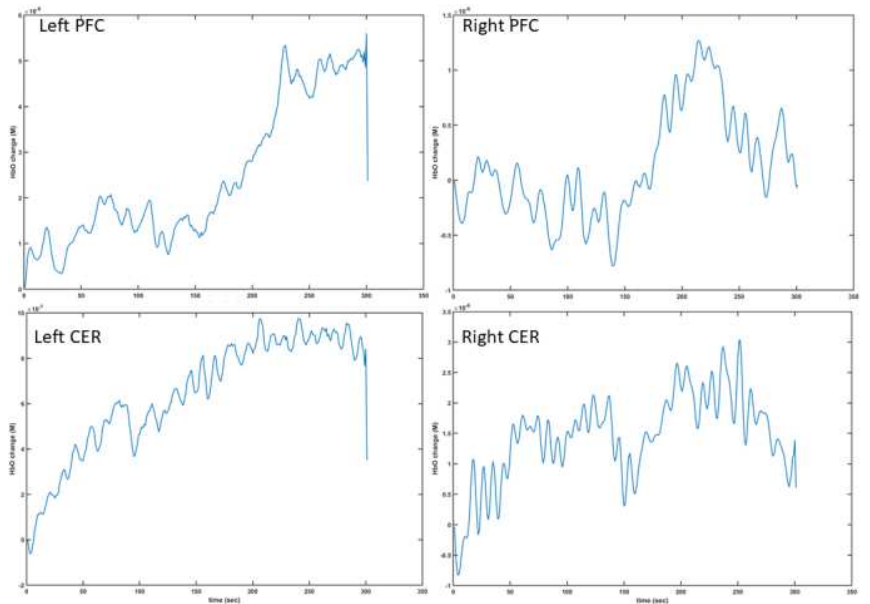

Fig. 5: $\mathrm{HbO}$ response of an illustrative subject at the bilateral prefrontal cortex (PFC) and the cerebellum (CER) due to $2 \mathrm{~mA}$ ctDCS targeting right cerebellar lobules VI-CrusI/II-VIIb (nonmotor representations).
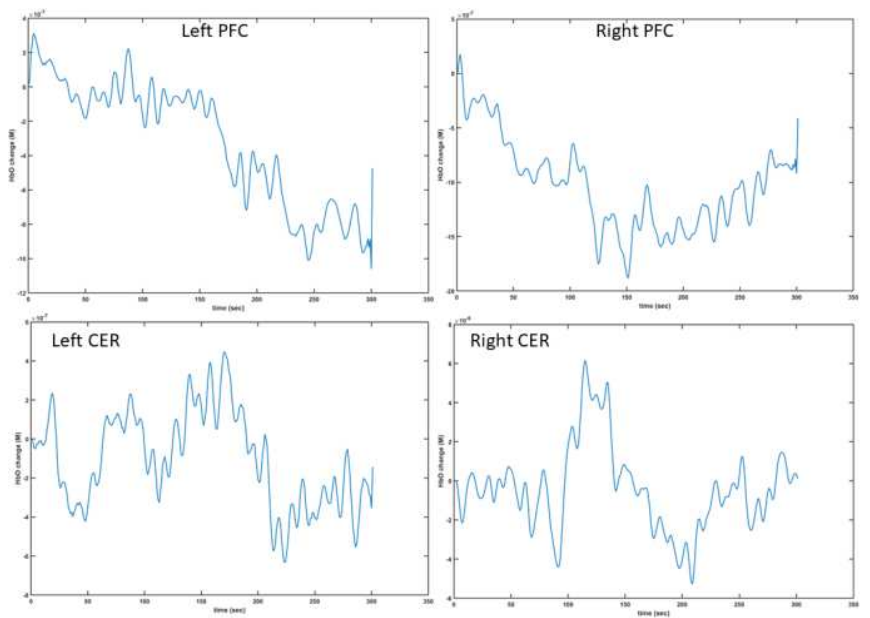

Fig. 6: $\mathrm{HbO}$ response of an illustrative subject at the bilateral prefrontal cortex (PFC) and the cerebellum (CER) due to $2 \mathrm{~mA}$ ctACS targeting right cerebellar lobules VI-CrusI/II-VIIb (nonmotor representations). 


\section{DISCUSSION}

In this study on feasibility testing of fNIRS of cerebellum and PFC, we found $\mathrm{HbO}$ response to ctDCS and ctACS using the same optimized montage (targeting right lobules VICrusI/II-VIIb) that were found to be different. Specifically, $2 \mathrm{~mA}$ ctDCS evoked similar $(\alpha=0.01) \mathrm{HbO}$ response across cerebellum and PFC brain regions that may be related to the modulation of Purkinje cells as well as deep cerebellar nuclei (see Fig. 3d) [4]. Indeed, tDCS can have effects on different cell populations in the cerebellar cortex that together will generate the effect which will be difficult to predict [22]. However, ctACS at the theta band frequency can increase the inhibitory tone that the cerebellum exerts over cerebrum due to selective recruitment of cerebellar granule cells and Golgi cells [6]. This modulation of the parallel fibre-Purkinje cell can lead to the modulation of the $\mathrm{HbO}$ activity at PFC that is then driving the ctACS via a phase amplitude coupling. This recurrent network can be driven to resonance that can be shown in simulation [23]. Here, adequate lag in the phase amplitude coupling may be necessary that can be elucidated from $\mathrm{HbO}$ time series from the cerebellum and PFC. In this preliminary study, we did not implement subject-specific lag for fNIRS-driven ctACS; however, we still found $\mathrm{HbO}$ change to be lower at the left PFC than the right PFC (see right panel of Fig. 7) during ctACS of right lobules VI-CrusI/II-VIIb ( $\mathrm{HbO}$ change higher in right than left cerebellum) indicating the recruitment of the cortico-cerebellothalamo-cortical loop.

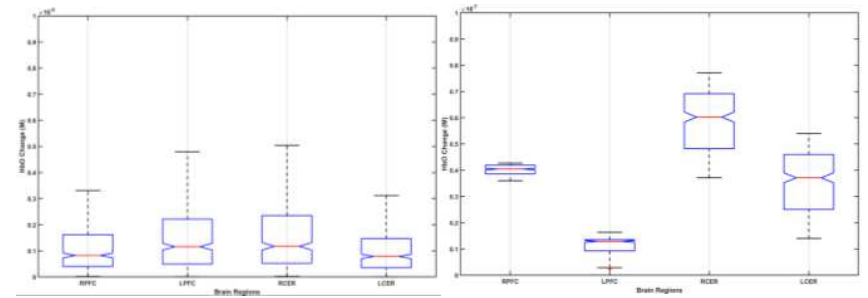

Fig. 7: Box-plot of post-intervention $\mathrm{HbO}$ change from preintervention baseline due to $2 \mathrm{~mA} c t D C S$ (left panel) and $2 \mathrm{~mA} c t A C S$ (right panel).

\section{REFERENCES}

[1] D. Solanki, Z. Rezaee, A. Dutta, and U. Lahiri, "Investigating the feasibility of cerebellar transcranial direct current stimulation to facilitate post-stroke overground gait performance in chronic stroke: a partial least-squares regression approach," Journal of NeuroEngineering and Rehabilitation, vol. 18, no. 1, p. 18, Jan. 2021, doi: 10.1186/s12984-021-00817-3.

[2] Z. Rezaee et al., "Feasibility of combining functional near-infrared spectroscopy with electroencephalography to identify chronic stroke responders to cerebellar transcranial direct current stimulation-a computational modeling and portable neuroimaging methodological study," Cerebellum, Mar. 2021, doi: 10.1007/s12311-021-01249-4.

[3] S. B. Zandvliet, C. G. M. Meskers, G. Kwakkel, and E. E. H. van Wegen, "Short-Term Effects of Cerebellar tDCS on Standing Balance Performance in Patients with Chronic Stroke and Healthy Age-Matched Elderly," Cerebellum, vol. 17, no. 5, pp. 575-589, Oct. 2018, doi: 10.1007/s12311-018-0939-0.

[4] Z. Rezaee et al., "Deep Cerebellar Transcranial Direct Current Stimulation of the Dentate Nucleus to Facilitate Standing Balance in Chronic Stroke Survivors-A Pilot Study," Brain Sciences, vol. 10, no. 2, p. 94, Feb. 2020, doi: 10.3390/brainsci10020094.

[5] S. Marek et al., "Spatial and Temporal Organization of the Individual Human Cerebellum," Neuron, vol. 100, no. 4, pp. 977-993.e7, Nov. 2018, doi: 10.1016/j.neuron.2018.10.010.

[6] D. Spampinato, E. Avci, J. Rothwell, and L. Rocchi, "Frequencydependent modulation of cerebellar excitability during the application of non-invasive alternating current stimulation," Brain
Stimul, vol. 14, no. 2, pp. 277-283, Apr. 2021, doi 10.1016/j.brs.2021.01.007.

[7] Z. Rezaee and A. Dutta, "Cerebellar Lobules Optimal Stimulation (CLOS): A Computational Pipeline to Optimize Cerebellar LobuleSpecific Electric Field Distribution," Front. Neurosci., vol. 13, 2019, doi: 10.3389/fnins.2019.00266.

[8] Y. Huang, A. Datta, M. Bikson, and L. C. Parra, "Realistic volumetric-approach to simulate transcranial electric stimulationROAST - a fully automated open-source pipeline," J. Neural Eng., vol. 16, no. 5, p. 056006, Jul. 2019, doi: 10.1088/1741-2552/ab208d.

[9] G. B. Saturnino, O. Puonti, J. D. Nielsen, D. Antonenko, K. H. H Madsen, and A. Thielscher, "SimNIBS 2.1: A Comprehensive Pipeline for Individualized Electric Field Modelling for Transcranial Brain Stimulation," bioRxiv, p. 500314, Dec. 2018, doi $10.1101 / 500314$.

[10] X. Guell and J. Schmahmann, "Cerebellar Functional Anatomy: a Didactic Summary Based on Human fMRI Evidence," Cerebellum, vol. 19, no. 1, pp. 1-5, Feb. 2020, doi: 10.1007/s12311-019-010839.

[11] R. L. Buckner, F. M. Krienen, A. Castellanos, J. C. Diaz, and B. T. T. Yeo, "The organization of the human cerebellum estimated by intrinsic functional connectivity," J Neurophysiol, vol. 106, no. 5, pp. 2322-2345, Nov. 2011, doi: 10.1152/jn.00339.2011.

[12] F. M. Krienen and R. L. Buckner, "Segregated fronto-cerebellar circuits revealed by intrinsic functional connectivity," Cereb. Cortex, vol. 19, no. 10, pp. 2485-2497, Oct. 2009, doi 10.1093/cercor/bhp135.

[13] M. A. Nitsche and W. Paulus, "Sustained excitability elevations induced by transcranial DC motor cortex stimulation in humans," Neurology, vol. 57, no. 10, pp. 1899-1901, Nov. 2001, doi: 10.1212/wnl.57.10.1899.

[14] C. Paquette, M. Sidel, B. A. Radinska, J.-P. Soucy, and A. Thiel, "Bilateral transcranial direct current stimulation modulates activation-induced regional blood flow changes during voluntary movement," J Cereb Blood Flow Metab, vol. 31, no. 10, pp. 20862095, Oct. 2011, doi: 10.1038/jcbfm.2011.72.

[15] "Grey-box modeling and hypothesis testing of functional nearinfrared spectroscopy-based cerebrovascular reactivity to anodal high-definition tDCS in healthy humans," Feb. 17, 2021 https://www.researchsquare.com (accessed Mar. 01, 2021).

[16] C. M. Aasted et al., "Anatomical guidance for functional nearinfrared spectroscopy: AtlasViewer tutorial," Neurophotonics, vol. 2, no. 2, Apr. 2015, doi: 10.1117/1.NPh.2.2.020801.

[17] D. Guhathakurta and A. Dutta, "Computational Pipeline for NIRSEEG Joint Imaging of tDCS-Evoked Cerebral Responses-An Application in Ischemic Stroke," Front. Neurosci., vol. 10, 2016, doi: 10.3389/fnins.2016.00261.

[18] G. A. Zimeo Morais, J. B. Balardin, and J. R. Sato, "fNIRS Optodes' Location Decider (fOLD): a toolbox for probe arrangement guided by brain regions-of-interest," Scientific Reports, vol. 8, no. 1, Art. no. 1, Feb. 2018, doi: 10.1038/s41598-018-21716-z.

[19] T. J. Huppert, S. G. Diamond, M. A. Franceschini, and D. A. Boas, "HomER: a review of time-series analysis methods for near-infrared spectroscopy of the brain,” Appl Opt, vol. 48, no. 10, pp. D280-D298, Apr. 2009.

[20] S. Jahani, S. K. Setarehdan, D. A. Boas, and M. A. Yücel, "Motion artifact detection and correction in functional near-infrared spectroscopy: a new hybrid method based on spline interpolation method and Savitzky-Golay filtering," Neurophotonics, vol. 5, no. 1, Jan. 2018, doi: 10.1117/1.NPh.5.1.015003.

[21] J. C. Ye, S. Tak, K. E. Jang, J. Jung, and J. Jang, "NIRS-SPM statistical parametric mapping for near-infrared spectroscopy," Neuroimage, vol. 44, no. 2, pp. 428-447, Jan. 2009, doi: 10.1016/j.neuroimage.2008.08.036.

[22] G. Batsikadze et al., "Effects of cerebellar transcranial direct current stimulation on cerebellar-brain inhibition in humans: A systematic evaluation," Brain Stimulation: Basic, Translational, and Clinical Research in Neuromodulation, vol. 0, no. 0, Apr. 2019, doi: 10.1016/j.brs.2019.04.010.

[23] A. Dutta, A. Ghosh, and S. Singh, "Deep Cerebellar Transcranial Electrical Stimulation: Hypothesis and Theory for Cannabis Use Disorder," Dec. 2020, doi: 10.20944/preprints202012.0178.v1. 